\title{
Momentum Kicks in Imperfect Which-Way Measurement
}

\author{
Neha Pathania and Tabish Qureshi
}

Centre for Theoretical Physics, Jamia Millia Islamia, New Delhi, India.

There has been an intense debate on the question as to whether a quanton, passing through a double-slit, experiences a 'momentum kick' due to the act of which-way detection. There have been conflicting points of view on this issue over many decades. This issue is addressed here in the general setting where the which-way detection may be imperfect. It is shown here that the loss of interference may still be interpreted as arising out of tiny momentum kicks which the quanton appears to receive, irrespective of the nature of the whichway detector. Interestingly, the magnitude of the random momentum kicks is always $h / 2 d, d$ being the slit separation, irrespective of how perfect or imperfect the which-way detection is. This is contrary to what has been suggested in the earlier literature. The imperfection of which-way detection decides how frequent are the momentum kicks. It has been shown earlier that for perfect which-way detection, the quanton receives a momentum kick fifty percent of the time. Here it is shown that for imperfect which-way detection, the quanton receives momentum kicks of the same magnitude, but less often. A precise relation between the frequency of kicks and the visibility of interference is found here.

\section{Introduction}

The concept of complementarity, introduced by Niels Bohr [1], is best illustrated in the two-slit experiment with particles. Right at the time of the formulation of Bohr's principle, Einstein proposed his famous recoilingslit experiment, in a bid to refute it [2]. Although Einstein's bid turned out to be unsuccessful, it generated a lively debate which continues to this day. With the advancement of technology and sophisticated experimental techniques, this thought experiment has now been realized in different ways [3-5]. Bohr invoked Heisenberg's uncertainty principle to give a rebuttal to Einstein by pointing out that measuring the momentum of the recoiling slit, in order to find which of the two slits the particle went through, would produce an uncertainty in the position of the recoiling slit, which in turn would wash out the interference. Bohr's reply led many authors to assume that complementarity was grounded in the uncertainty principle, and was probably another Neha Pathania: neha@ctp-jamia.res.in Tabish Qureshi: tabish@ctp-jamia.res.in

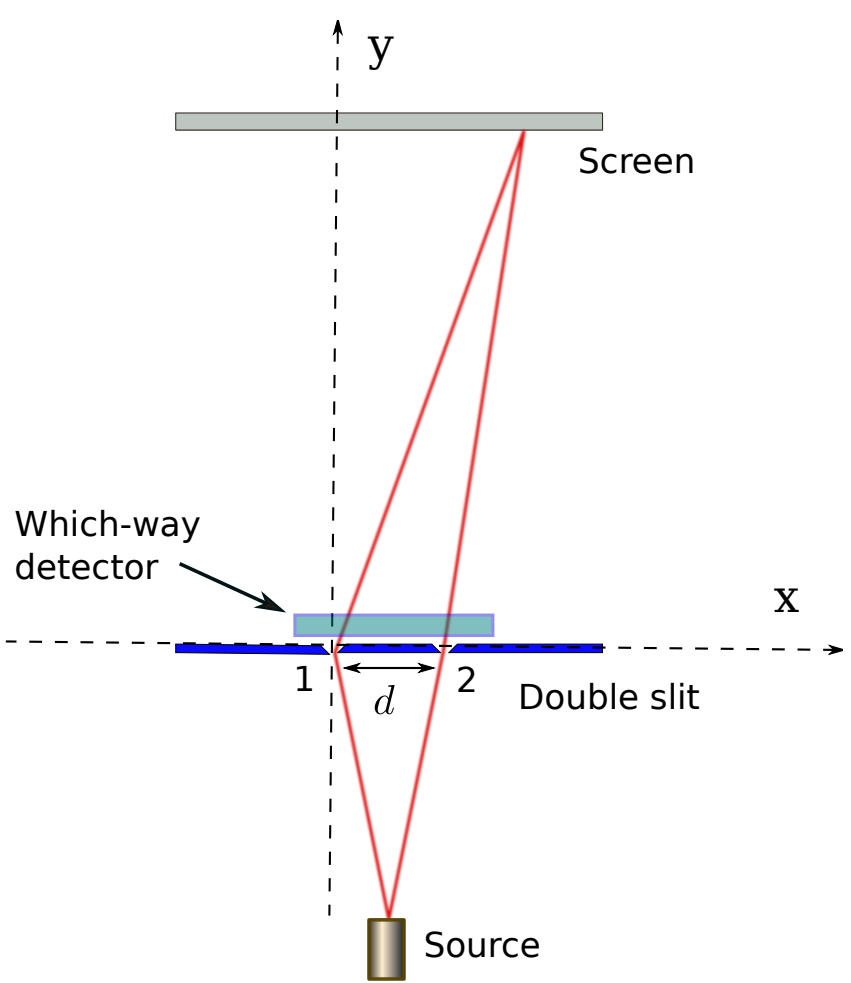

Figure 1: Schematic diagram of a two-slit interference experiment in the presence of a which-way detector. Slit 1 is located at $x=0$ and slit 2 is located at $x=d$. The quanton travels in the positive $y$ direction.

way of stating it. Such belief prevailed until Scully, Englert and Walther proposed a which-way experiment with atoms and using micromaser cavities as a which-way detector. They claimed that the loss of interference in the proposed experiment was purely a result of quantum correlations between the path states of the atom and the states of cavities, and did not involve any positionmomentum uncertainty [6]. They concluded that the which-way detection process does not involve any momentum transfer to the interfering particle. Storey et.al. produced a counter argument to this claim by apparently proving that if an interference pattern is destroyed in a which-way experiment, a momentum of at least the magnitude $\hbar / d$ should be transferred to the particle, where $d$ is the separation between the two slits [7]. A momentum transfer of an amount smaller than that would not destroy the interference completely, they argued. The focus of the debate then shifted to settling the question whether there is a momentum transfer to the particle involved in the process of which-way detection [8-13]. 
Later it was shown that the complementarity principle can be understood in terms of the ubiquitous entanglement between the particle and the which-way detector, and also equivalently in terms of the uncertainty between certain operators of the which-way detector, and the well-known wave-particle duality relation [14] can be derived from both $[15,16]$. However, the question whether there is a momentum transfer to the particle or not, is still being actively studied $[17,18]$. Very recently it was shown that the two views which say that there is, and there isn't a momentum kick, are completely equivalent, and depend on which basis set of the which-way detector one is considering [19]. However, perfect which-way detection was used in that argument. In situations where the which-way detection may not be perfect, can one still talk of momentum kicks as shown in the earlier result [19]? That is the question we address in the following investigation.

\section{Imperfect which-way detection and interference}

Let us consider a quanton passing through a doubleslit, as shown in Figure 1. The traveling quanton also interacts with a which-way detector, the detailed nature of which we do not specify. We write the joint state of the quanton and which-way detector as the state which was consider by Storey et. al. [7]

$$
|\Psi\rangle=\int d x \psi(x)|x\rangle \otimes\left|D_{x}\right\rangle,
$$

where $\psi(x)$ represents the wavefunction of the quanton just as it emerges from the double-slit, and $\left|D_{x}\right\rangle$ are the states of the which-way detector. Instead of following the general analysis of Storey et. al., we use the fact that two slits are physically separated, and the wavefunctions localized at each, are disjoint, and orthogonal to each other. Thus we can write

$$
|\Psi\rangle=\frac{1}{\sqrt{2}} \int d x\left[\psi_{1}(x)\left|d_{1}\right\rangle+\psi_{2}(x)\left|d_{2}\right\rangle\right]|x\rangle,
$$

where are $\psi_{1}(x), \psi_{2}(x)$ are wavefunctions sharply localized at $x=0$ and $x=d$, respectively, and are orthogonal by virtue of their spatial separation. We also recognize the fact the which-way detector states $\left|d_{1}\right\rangle,\left|d_{2}\right\rangle$ may or may not have a position degree of freedom. For example, they could just represent spin degree of freedom. Consequently $\left|d_{1}\right\rangle,\left|d_{2}\right\rangle$ are assumed to be certain normalized states of the which-way detector, in an effectively twodimensional Hilbert space. If $\left|d_{1}\right\rangle,\left|d_{2}\right\rangle$ are orthogonal, they will perform a perfect which-way detection of the quanton. Here we assume that $\left|d_{1}\right\rangle,\left|d_{2}\right\rangle$ are not necessarily orthogonal. For our purpose, we omit the integral in (2), and write the combined state as a function of $x$

$$
|\Psi(x)\rangle=\frac{1}{\sqrt{2}}\left[\psi_{1}(x)\left|d_{1}\right\rangle+\psi_{2}(x)\left|d_{2}\right\rangle\right],
$$

After emerging from the double slit, the quanton travels a distance $D$ to the screen in a time $t$, and the state at time $t$ is given by

$$
\Psi(x, t)=\frac{1}{\sqrt{2}} \boldsymbol{U}(t)\left[\psi_{1}(x)\left|d_{1}\right\rangle+\psi_{2}(x)\left|d_{2}\right\rangle\right],
$$

where $\boldsymbol{U}(t)$ represents the Schrödinger time evolution operator. A full treatment would require taking into account the $\mathrm{y}$-dependence of the wavefunction $\Psi(x, t)$. However, the evolution in the $y$-direction is rather trivial, and serves only to transport the quanton from the slits to the screen. It is independent of the evolution in the $\mathrm{x}$-direction, and thus plays no role in the dynamics of interference. For this reason we choose to ignore the explicit $y$-dependence of the quanton wavefunction, and just assume that $\Psi(x, t)$ is the wavefunction of the quanton at time $t$, when it has reached the screen. The probability density of the quanton falling on the screen at a position $x$, is then given by

$$
\begin{aligned}
|\Psi(x, t)|^{2}= & \frac{1}{2}\left[\left|\psi_{1}(t)\right|^{2}+\left|\psi_{2}(t)\right|^{2}\right. \\
& \left.+\psi_{1}^{*}(t) \psi_{2}(t)\left\langle d_{1} \mid d_{2}\right\rangle+\psi_{2}^{*}(t) \psi_{1}(t)\left\langle d_{2} \mid d_{1}\right\rangle\right],
\end{aligned}
$$

where $\psi_{j}(t) \equiv \psi_{j}(x, t)=\boldsymbol{U}(t) \psi_{j}(x)$. Notable is the fact that the last two terms in the above equation, which represent interference, are reduced by a factor $\left|\left\langle d_{1} \mid d_{2}\right\rangle\right|$. In fact, it is well known that in a symmetric two slit interference experiment, the visibility of interference turns out to be $[14,15,20]$

$$
\mathcal{V}=\left|\left\langle d_{1} \mid d_{2}\right\rangle\right|
$$

Now if there were no which-way detector in the path of the quanton, its wavefunction would be given by

$$
\Psi_{0}(x)=\frac{1}{\sqrt{2}}\left[\psi_{1}(x)+\psi_{2}(x)\right],
$$

which would lead to maximum visibility $\mathcal{V}=1$. It should be emphasized here that one can, in principle, choose an interaction between the quanton and the which-way detector such that the $\psi_{1}(x), \psi_{2}(x)$ in (7) are the same as those in (3), and the which-way detection does not change the individual states of the particle emerging from the slits. So there is no question of any additional momentum kick or 'momentum back-action' which has been considerably debated in the literature. This was the point of view of Scully, Englert and Walther [6].

\section{Distinguishing between the two paths}

If the states $\left|d_{1}\right\rangle,\left|d_{2}\right\rangle$ in (3) are orthogonal, the two paths can be distinguished unambiguously, by simply measuring an observable of the which-way detector for which $\left|d_{1}\right\rangle,\left|d_{2}\right\rangle$ yield different eigenvalues. However, if $\left|d_{1}\right\rangle,\left|d_{2}\right\rangle$ are not orthogonal, no such observable exists. However, there is a method in which two non-orthogonal states can be distinguished unambiguously, with the 
caveat that the method can occasionally fail. The success and failure events are distinct, and one knows if one has failed or not. The result is that one knows the events in which one succeeds, and in those, one can distinguish between the two non-orthogonal state unambiguously. This procedure is called unambiguous quantum state discrimination (UQSD) [21-24].

There are different ways of doing UQSD. In one method it is assumed that the Hilbert space of the nonorthogonal states $\left|d_{1}\right\rangle,\left|d_{2}\right\rangle$ (path-detector states in our case) is not two-dimensional, but three dimensional, described by an orthonormal basis of states $\left|q_{1}\right\rangle,\left|q_{2}\right\rangle,\left|q_{3}\right\rangle$. The reason for choosing this method will become clear in the following analysis. The basis is chosen in such a way that the detector states $\left|d_{1}\right\rangle,\left|d_{2}\right\rangle$ can be represented as [24]

$$
\begin{aligned}
& \left|d_{1}\right\rangle=\alpha\left|q_{1}\right\rangle+\beta\left|q_{3}\right\rangle \\
& \left|d_{2}\right\rangle=\gamma\left|q_{2}\right\rangle+\delta\left|q_{3}\right\rangle
\end{aligned}
$$

where $\alpha$ and $\gamma$ are real, and $\beta, \delta$ satisfy

$$
\begin{aligned}
|\beta||\delta| & \geq\left|\left\langle d_{1} \mid d_{2}\right\rangle\right|, \\
|\beta|^{2} & =\left|\left\langle d_{1} \mid d_{2}\right\rangle\right| \\
\alpha=\gamma & =\sqrt{1-\left|\left\langle d_{1} \mid d_{2}\right\rangle\right|}
\end{aligned}
$$

In the expanded Hilbert space, one can now measure an operator (say)

$$
\boldsymbol{Q}=a\left|q_{1}\right\rangle\left\langle q_{1}|+b| q_{2}\right\rangle\left\langle q_{2}|+c| q_{3}\right\rangle\left\langle q_{3}\right| .
$$

Here $\left\langle q_{2} \mid d_{1}\right\rangle=0$ and $\left\langle q_{1} \mid d_{2}\right\rangle=0$, which means that getting a measured eigenvalue $a$ of $Q$ means that the state could not have been $\left|d_{2}\right\rangle$, and thus it is $\left|d_{1}\right\rangle$. Similarly, getting a measured eigenvalue $b$ of $\boldsymbol{Q}$ means that the state could not have been $\left|d_{1}\right\rangle$, and thus it is $\left|d_{2}\right\rangle$. In these two cases one can unambiguously distinguish between $\left|d_{1}\right\rangle$ and $\left|d_{2}\right\rangle$. However, $\left\langle q_{3} \mid d_{1}\right\rangle \neq 0$ and $\left\langle q_{3} \mid d_{2}\right\rangle \neq 0$ in which case one cannot tell if the state was $\left|d_{1}\right\rangle$ or $\left|d_{2}\right\rangle$. In order to make the procedure maximally efficient, one would like to minimize the probability of getting the eigenvalue $c$, which represents the failure of the state discrimination. The values of $\beta, \delta$ that we have chosen in (9) are such that they minimize the probability of failure, and maximize the probability of successfully distinguishing between $\left|d_{1}\right\rangle$ and $\left|d_{2}\right\rangle$ [24].

Next we substitute (8) in (3) to write

$$
\begin{aligned}
|\Psi(x)\rangle= & \frac{1}{\sqrt{2}}\left[\psi_{1}(x)\left(\alpha\left|q_{1}\right\rangle+\beta\left|q_{3}\right\rangle\right)\right. \\
& \left.+\psi_{2}(x)\left(\alpha\left|q_{2}\right\rangle+\delta\left|q_{3}\right\rangle\right)\right], \\
= & \frac{1}{\sqrt{2}} \alpha\left[\psi_{1}(x)\left|q_{1}\right\rangle+\psi_{2}(x)\left|q_{2}\right\rangle\right] \\
& +\frac{1}{\sqrt{2}}\left[\beta \psi_{1}(x)+\delta \psi_{2}(x)\right]\left|q_{3}\right\rangle .
\end{aligned}
$$

We would like to emphasize that by writing the state in the above form, we have neither changed anything, nor carried out any physical process. From (9), one can see that $\delta$ is equal to $\beta$ up to a phase factor like $e^{i \theta}$. Let us first assume $\theta$ to be zero, so that the state (11) simplifies to

$$
\begin{aligned}
|\Psi(x)\rangle= & \frac{\alpha}{\sqrt{2}}\left[\psi_{1}(x)\left|q_{1}\right\rangle+\psi_{2}(x)\left|q_{2}\right\rangle\right] \\
& +\frac{\beta}{\sqrt{2}}\left[\psi_{1}(x)+\psi_{2}(x)\right]\left|q_{3}\right\rangle .
\end{aligned}
$$

We introduce two new states $\left|q_{ \pm}\right\rangle=\frac{1}{\sqrt{2}}\left(\left|q_{1}\right\rangle \pm\left|q_{2}\right\rangle\right)$, in terms of which the state (12) can be written as

$$
\begin{aligned}
|\Psi(x)\rangle= & \frac{\alpha}{2}\left[\psi_{1}(x)+\psi_{2}(x)\right]\left|q_{+}\right\rangle \\
& +\frac{\alpha}{2}\left[\psi_{1}(x)-\psi_{2}(x)\right]\left|q_{-}\right\rangle \\
& +\frac{\beta}{\sqrt{2}}\left[\psi_{1}(x)+\psi_{2}(x)\right]\left|q_{3}\right\rangle .
\end{aligned}
$$

It is interesting to observe that the state of the quanton correlated to the which-way detector state $\left|q_{+}\right\rangle$and $\left|q_{3}\right\rangle$ is the same, and is simply the state of the quanton that would have been, if there were no which-way detector, i.e., $\left|\Psi_{0}(x)\right\rangle$ given by (7). The quanton state correlated to $\left|q_{-}\right\rangle$differs from $\left|\Psi_{0}(x)\right\rangle$ only in that the two paths are out of phase by $\pi$.

\section{Apparent momentum kick}

When the quanton emerges from the double-slit, the state consists of two parts each localized within the very narrow region of the slit. Thus $\psi_{1}(x)$ represents a state sharply localized at $x=0$ (position of slit 1 ), and $\psi_{2}(x)$ is sharply localized at $x=d$ (position of slit 2). The width of these two states in position would be approximately the same as the width of each slit. Of course, after emerging from the slits, when $\psi_{1}(x)$ and $\psi_{2}(x)$ evolve in time, their widths will increase very fast. At the double slit, the quanton state correlated to $\left|q_{-}\right\rangle$can be written in a slightly different way:

$$
\frac{1}{\sqrt{2}}\left[\psi_{1}(x)-\psi_{2}(x)\right]=\frac{1}{\sqrt{2}} \exp \left(\frac{i}{\hbar} p_{0} x\right)\left[\psi_{1}(x)+\psi_{2}(x)\right],
$$

where $p_{0}=h / 2 d$. In arriving at (14), we have assumed that $\psi_{1}(x)$ has support only in a narrow region localized at $x=0$, and $\psi_{2}(x)$ has support only in a narrow region localized at $x=d$. The exponential function $\exp \left(\frac{i}{\hbar} p_{0} x\right)$ in these two regions can be approximated by $\exp \left(\frac{i}{\hbar} p_{0} \cdot 0\right)=1$ and $\exp \left(\frac{i}{\hbar} p_{0} d\right)=e^{i \pi}=-1$, respectively. The factor $e^{\frac{i}{\hbar} p_{0} x}$ can be considered as a momentum kick that the quanton experiences, in comparison to the quanton state correlated to $\left|q_{+}\right\rangle$, which also happens to be identical to the undisturbed state $\left|\Psi_{0}(x)\right\rangle$. The state (13) can now be written as

$$
\begin{aligned}
|\Psi(x)\rangle= & \frac{\alpha}{2}\left[\psi_{1}(x)+\psi_{2}(x)\right]\left|q_{+}\right\rangle \\
& +\frac{\alpha}{2} e^{\frac{i}{\hbar} p_{0} x}\left[\psi_{1}(x)+\psi_{2}(x)\right]\left|q_{-}\right\rangle \\
& +\frac{\beta}{\sqrt{2}}\left[\psi_{1}(x)+\psi_{2}(x)\right]\left|q_{3}\right\rangle \\
= & \Psi_{0}(x)\left[\frac{\alpha}{\sqrt{2}}\left|q_{+}\right\rangle+\beta\left|q_{3}\right\rangle\right]+e^{\frac{i}{\hbar} p_{0} x} \Psi_{0}(x) \frac{\alpha}{\sqrt{2}}\left|q_{-}\right\rangle
\end{aligned}
$$

Given the fact that $\left|q_{+}\right\rangle,\left|q_{-}\right\rangle,\left|q_{3}\right\rangle$ form an orthonormal set, equation (15) implies that the quanton passes undisturbed whenever the which-way detector state is $\left|q_{+}\right\rangle$or 
$\left|q_{3}\right\rangle$. But when the which-way detector state is $\left|q_{-}\right\rangle$, the quanton appears to experience a momentum kick of magnitude $p_{0}=h / 2 d$. Let us see how the state (15) looks in the momentum basis. It is straightforward to write

$$
\Phi(p)=\Phi_{0}(p)\left[\frac{\alpha}{\sqrt{2}}\left|q_{+}\right\rangle+\beta\left|q_{3}\right\rangle\right]+\Phi_{0}\left(p-p_{0}\right) \frac{\alpha}{\sqrt{2}}\left|q_{-}\right\rangle,
$$

where $\Phi_{0}(p)$ is the momentum representation of the undisturbed quanton state $\Psi_{0}(x)$, given by (7). This form clearly brings out the apparent momentum kick of magnitude $p_{0}$. Looking at it, one realizes that the mistake Storey et. al. [7] made was in forcing the which-way detector to have position degree of freedom, and then misinterpreting the momentum shift in the quanton as a momentum transfer from the path detector to the quanton. The apparent moment shift is just an artifact of considering the which-way detector in a different basis. A similar mistake led others [10] to conclude that it is not possible to demonstrate momentum kicks in the proposed experiment of Scully, Englert and Walther [6], because the microwave cavity which-way detectors, in their setup, did not involve any position degrees of freedom. We have demonstrated here that the concept of momentum kicks is very general and works for any kind of which-way detector.

For imperfect which-way detection, Storey et. al. had derived a relation between the interference visibility and the maximum transferred momentum $p_{m}$ [7]

$$
\frac{p_{m} d}{\hbar} \geq 1-\mathcal{V}
$$

This gives the impression that the magnitude of momentum kicks will become smaller if the which-way detection is imperfect. However, the preceding analysis shows that the magnitude of the momentum kicks is $h / 2 d$, and does not depend on the how good the which-way detection is. So what does depend on the efficiency of which-way detection? Equation (16) says that the probably of the quanton getting a momentum kick is $\alpha^{2} / 2$. Remembering the value of $\alpha$ from (9), we can write the probability of a quanton getting a kick, or the fraction of quantons (from a stream) which receive a momentum kick, as

$$
F_{k}=\frac{1}{2}\left(1-\left|\left\langle d_{1} \mid d_{2}\right\rangle\right|\right) .
$$

For perfect which-way detection $F_{k}=\frac{1}{2}$ which agrees with the earlier result [19]. If the which-way detection becomes more imperfect, the quanton receives apparent momentum kicks of the same magnitude, but less often. The probability of the quanton receiving momentumkicks is also related to the visibility of interference in a straightforward manner

$$
F_{k}=\frac{1}{2}(1-\mathcal{V})
$$

So, the interference visibility is not related to the magnitude of the momentum kicks, but to the probability of the quanton receiving a momentum kick.
Let us now consider the case where $\theta$ may not be zero, and $\delta=e^{i \theta} \beta$. In this case, instead of (13) one would write

$$
\begin{aligned}
|\Psi(x)\rangle= & \frac{\alpha}{2}\left[\psi_{1}(x)+\psi_{2}(x)\right]\left|q_{+}\right\rangle \\
& +\frac{\alpha}{2}\left[\psi_{1}(x)-\psi_{2}(x)\right]\left|q_{-}\right\rangle \\
& +\frac{\beta}{\sqrt{2}}\left[\psi_{1}(x)+e^{i \theta} \psi_{2}(x)\right]\left|q_{3}\right\rangle .
\end{aligned}
$$

The question one may then ask is, can the factor $e^{i \theta}$ be also interpreted as a momentum kick, and does it affect the visibility of interference? It is true that the last term may also be written as

$$
\begin{aligned}
|\Psi(x)\rangle= & \frac{\alpha}{2}\left[\psi_{1}(x)+\psi_{2}(x)\right]\left|q_{+}\right\rangle \\
& +\frac{\alpha}{2}\left[\psi_{1}(x)-\psi_{2}(x)\right]\left|q_{-}\right\rangle \\
& +\frac{\beta}{\sqrt{2}} e^{\frac{i}{\hbar} p_{e} x}\left[\psi_{1}(x)+\psi_{2}(x)\right]\left|q_{3}\right\rangle,
\end{aligned}
$$

where $p_{e}=\theta \hbar / d$ may be interpreted as the magnitude of a momentum kick. However, it is easy to see that it will not affect the interference visibility. The two interference patterns arising from the first two terms in (21) cancel each other out exactly, and the effect of $e^{\frac{i}{\hbar} p_{e} x}$ on the third interference will be to just shift it by a fixed amount. While it is clear that the presence of $e^{\frac{i}{\hbar} p_{e} x}$ does not affect the interference visibility, it also highlights the rather artificial nature of the concept of momentum kicks. It would probably be better to look at it as a phase difference between the two paths $[25,26]$.

Lastly we probe a situation where the detector states $\left|q_{ \pm}\right\rangle$may form any general basis, which is unbiased with respect to $\left|q_{1}\right\rangle,\left|q_{2}\right\rangle$. For example, let us assume them to have the form $\left|q_{ \pm}\right\rangle=\frac{1}{\sqrt{2}}\left(\left|q_{1}\right\rangle \pm e^{i \theta}\left|q_{2}\right\rangle\right)$, where $\theta$ is an arbitrary angle. Using this basis, the state in (12) can be written as

$$
\begin{aligned}
|\Psi(x)\rangle= & \frac{\alpha}{2}\left[\psi_{1}(x)+e^{-i \theta} \psi_{2}(x)\right]\left|q_{+}\right\rangle \\
& +\frac{\alpha}{2}\left[\psi_{1}(x)-e^{-i \theta} \psi_{2}(x)\right]\left|q_{-}\right\rangle \\
& +\frac{\beta}{\sqrt{2}}\left[\psi_{1}(x)+\psi_{2}(x)\right]\left|q_{3}\right\rangle .
\end{aligned}
$$

Since the two quanton states correlated with $\left|q_{ \pm}\right\rangle$differ from each other only in a phase difference of $\pi$ between the two paths, it is straightforward to see that the state $\frac{1}{\sqrt{2}}\left[\psi_{1}(x)-e^{-i \theta} \psi_{2}(x)\right]$ is the same as $e^{\frac{i}{\hbar} p_{0} x} \frac{1}{\sqrt{2}}\left[\psi_{1}(x)+e^{-i \theta} \psi_{2}(x)\right]$, where $p_{0}=h / 2 d$ is a momentum kick. However, if one insists on calculating the momentum kicks with respect to the original state without any path detector, $\frac{1}{\sqrt{2}}\left[\psi_{1}(x)+\psi_{2}(x)\right]$, the momentum kicks come out to be $\hbar \theta / d$ and $\hbar \theta / d+h / 2 d$. So, there appears to be an ambiguity in the magnitude of the momentum kicks. However, notice that the momentum kick difference between the two states is still $h / 2 d$. Thus to avoid ambiguity, the momentum kicks should only be interpreted as relative kicks between the two states of the quanton. This aspect is easily understood by recalling that in the phenomenon of quantum erasure, the recovered interference may be shifted from the center, depending on which basis of the which-way detector one is using [27]. 


\section{Conclusion}

With the aim of clarifying the long-standing controversial issue of momentum kicks in which-way detection, we have theoretically analyzed a two-slit interference experiment with imperfect which-way detection, using the concept of UQSD. We have shown that the partial loss of interference may be interpreted as arising due to random momentum kicks which the quanton appears to experience. Interestingly, the relative magnitude of the momentum kicks always remains the same, $h / 2 d$, irrespective of the efficiency of which-way detection. Earlier arguments seemed to suggest otherwise [7]. However, as the which-way detection becomes more imperfect, the momentum kicks become less frequent. In the case of perfect which-way detection, the quanton experiences momentum kicks fifty percent of the time.

While the analysis presented here uses UQSD only as a tool to clarify the concept of momentum kicks, all this can be easily experimentally tested, as unambiguous path discrimination in two-path interference has already been experimentally demonstrated $[28,29]$.
The lesson learnt here is that the roots of complementarity lie in the ubiquitous entanglement between the quanton and the which-way detector. When the which-way detector is looked at in one particular basis, the loss of interference can be interpreted as arising from the quantum correlation between the two paths and two states of the which-way detector. If the which-way detector is looked at in another mutually unbiased basis, the loss of interference can be interpreted as arising from the quanton experiencing random momentum kicks of magnitude $h / 2 d$. However, it should be emphasized that these are only apparent momentum kicks arising out of a phase difference between the two paths. There is no real momentum transfer to the quanton from anywhere. We hope this finally puts the controversy surrounding the issue of momentum kicks at rest.

\section{Acknowledgements}

Neha Pathania acknowledges financial support from the Department of Science and Technology, through the Inspire Fellowship (registration number IF180414).

\section{References}

[1] N. Bohr, "The quantum postulate and the recent development of atomic theory," Nature (London) 121, 580-591 (1928).

[2] N. Bohr, in Albert Einstein: Philosopher-Scientist (ed. Schilpp, P. A.) 200-241 (Library of Living Philosophers, Evanston, 1949); reprinted in Quantum Theory and Measurement (eds J.A. Wheeler, W.H. Zurek,) 9-49 (Princeton Univ. Press, 1983).

[3] X-J Liu, Q. Miao, F. Gel'mukhanov, M. Patanen, O. Travnikova, C. Nicolas, H. Agren, K. Ueda, C. Miron "Einstein-Bohr recoiling double-slit gedanken experiment performed at the molecular level," $\mathrm{Na}$ ture Photonics 9, 120-125 (2015).

[4] L.Ph.H. Schmidt, J. Lower, T. Jahnke, S. Schößler, M.S. Schöffler, A. Menssen, C. Lévêque, N. Sisourat, R. Taïeb, H. Schmidt-Böcking, and R. Dörner, "Momentum Transfer to a Free Floating Double Slit: Realization of a Thought Experiment from the EinsteinBohr Debates," Phys. Rev. Lett. 111, 103201 (2013).

[5] R.S. Utter, J.M. Feagin, "Trapped-ion realization of Einstein's recoiling-slit experiment", Phys. Rev. A 75, 062105 (2007)

[6] M.O. Scully, B.G. Englert, H. Walther, "Quantum optical tests of complementarity," Nature 351,111116 (1991).

[7] E.P. Storey, S.M. Tan, M.J. Collett, D.F. Walls, "Path detection and the uncertainty principle," $\mathrm{Na}$ ture 367, 626-628, (1994).
[8] B.-G. Englert, M.O. Scully, H. Walther, "Complementarity and uncertainty," Nature 375, 367 (1995).

[9] E.P. Storey, S.M. Tan, M.J. Collett, D.F. Walls, "Complementarity and uncertainty," Nature 375 , 368 (1995)

[10] H. Wiseman, F. Harrison, "Uncertainty over complementarity?" Nature 377, 584 (1995).

[11] H.M. Wiseman, F.E. Harrison, M.J. Collett, S.M. Tan, D.F. Walls, R.B. Killip, "Nonlocal momentum transfer in welcher Weg measurements," Phys. Rev. A 56, 55 (1997)

[12] H.M. Wiseman, "Bohmian analysis of momentum transfer in welcher Weg measurements," Phys. Rev. A 581740 (1998).

[13] H. Wiseman, "Directly observing momentum transfer in twin-slit which-way experiments" Phys. Lett. A 311, 285 (2003).

[14] B-G. Englert, "Fringe visibility and which-way information: an inequality", Phys. Rev. Lett. 77, 2154 (1996).

[15] T. Qureshi, R. Vathsan, “Einstein's recoiling slit experiment, complementarity and uncertainty," Quanta 2, 58-65 (2013).

[16] S. Dürr, G. Rempe, "Can wave-particle duality be based on the uncertainty relation?" Am. J. Phys. 58, 1021-1024 (2000).

[17] R. Mir, J.S. Lundeen, M.W. Mitchell, A.M. Steinberg, J.L. Garretson, H.M. Wiseman, "A doubleslit 'which-way' experiment on the complementar- 
ity-uncertainty debate," New J. Phys. 9, 287 (2007).

[18] Y. Xiao, H.M. Wiseman, J-S. Xu, Y. Kedem, C-F. $\mathrm{Li}, \mathrm{G}-\mathrm{C}$. Guo, "Observing momentum disturbance in double-slit 'which-way' measurements" Sci. Adv. 5, eaav9547 (2019).

[19] T. Qureshi, "Which-way measurement and momentum kicks," EPL 123, 30007 (2018).

[20] K. Menon, T. Qureshi, "Wave-particle duality in asymmetric beam interference," Phys. Rev. A 82, 022130 (2018).

[21] I.D. Ivanovic, "How to differentiate between nonorthogonal states", Phys. Lett. A 123, 257 (1987).

[22] D. Dieks, "Overlap and distinguishability of quantum states," Phys. Lett. A 126, 303 (1988).

[23] A. Peres, "How to differentiate between nonorthogonal states ," Phys. Lett. A 128, 19 (1988).

[24] G. Jaeger, A. Shimony, "Optimal distinction between two non-orthogonal quantum states," Phys. Lett. A 197, 83 (1995).
[25] A. Luis, L.L. Sánchez-Soto, "Complementarity enforced by random classical phase kicks," Phys. Rev. Lett. 81, 4031 (1998).

[26] C.S. Unnikrishnan, "Origin of quantum-mechanical complementarity without momentum back action in atom-interferometry experiments," Phys. Rev. A 62, 015601 (2000)

[27] T. Qureshi, "The delayed-choice quantum eraser leaves no choice," Int. J. Theor. Phys. (2021)

[28] L. Neves, G. Lima, J. Aguirre, F. A. Torres-Ruiz, C. Saavedra, A. Delgado, "Control of quantum interference in the quantum eraser," New J. Phys. 11, 073035 (2009).

[29] Y. L. Len, J. Dai, B-G Englert, L.A. Krivitsky, “Unambiguous path discrimination in a two-path interferometer," Phys. Rev. A 98, 022110 (2018). 